\title{
Survival and stability of patients with urothelial cancer and spinal bone metastases after palliative radiotherapy
}

\author{
Robert Foerster ${ }^{1,3}$, Katharina Hees ${ }^{2}$, Thomas Bruckner ${ }^{2}$, Tilman Bostel ${ }^{1,3}$, \\ Ingmar Schlampp ${ }^{1,3}$, Tanja Sprave ${ }^{1,3}$, Nils H. Nicolay ${ }^{1,3}$, Juergen Debus ${ }^{1,3}$, Harald Rief ${ }^{1,3}$ \\ ${ }^{1}$ Department of Radiation Oncology, University Hospital Heidelberg, Heidelberg, Germany \\ 2 Department of Medical Biometry, University Hospital Heidelberg, Heidelberg, Germany \\ ${ }^{3}$ National Center for Radiation Research in Oncology (NCRO), Heidelberg Institute for Radiation Oncology (HIRO), \\ Heidelberg, Germany
}

Radiol Oncol 2018; 52(2): 189-194.

Correspondence to: Harald Rief, M.D., Ph.D., Department of Radiation Oncology, University Hospital Heidelberg, Im Neuenheimer Feld 400, 69120 Heidelberg, Germany. Phone: +49 622156 8202; Fax: +49 622156 5353; E-mail: harald.rief@gmx.at

Disclosure: No potential conflicts of interest were disclosed.

Background. The aim of the study was to analyze survival and stability of patients with urothelial cell cancer and spinal bone metastases (SBM) after radiotherapy (RT). Furthermore, to assess the effects of RT on bone mineral density (BMD) as a local response in SBM after RT.

Patients and methods. Survival of 38 patients with 132 SBM from urothelial cancer, treated from January 2000 to January 2012, was calculated. Stability of irradiated thoracic and lumbar SBM was retrospectively evaluated in computed tomography (CT) scans using the validated Taneichi et al. score. Difference in BMD, measured in Hounsfield units (HU), of the SBM before and at 3 and 6 months after RT was analyzed.

Results. All patients died during follow-up. Overall survival (OS) after 6 months, 1 year and 2 years was $90 \%, 80 \%$ and $40 \%$, respectively. Bone survival (BS) was $85 \%, 64 \%$ and $23 \%$ after 6 months, 1 year and 2 years, respectively. Survival from start of RT (RTS) was $42 \%$ after 6 months, $18 \%$ after 1 year and $5 \%$ after 2 years. Only $11 \%$ received bisphosphonates. Stability did not improve at 3 or 6 months after RT. BMD increased by $25.0 \mathrm{HU} \pm 49.7$ SD after 3 months $(p=0.001)$ and by $24.2 \mathrm{HU} \pm 52.2 \mathrm{SD}$ after 6 months ( $p=0.037)$. Pain relief ( $\geq 2$ points on the visual analogue scale) was achieved in only $27 \%$ of patients.

Conclusions. Benefit from palliative RT of painful or unstable SBM is limited in these patients and they should be carefully selected for RT. Shorter fractionation schedules may be preferred and outcome may improve with concomitant bisphosphonates.

Key words: bone density; bone metastases; stability; survival; urothelial cancer

\section{Introduction}

Approximately one quarter of patients with metastatic urothelial cancer present with bone metastases. ${ }^{1}$ Spinal bone metastases (SBM) are commonly associated with drug-resistant pain, pathological fractures and neurological complications, resulting in substantial morbidity and reduced quality of life (QoL).

Treatment of these patients requires an interdisciplinary approach and palliative radiotherapy
(RT) remains the most important treatment option, particularly for persistent pain, existing or impending instability and neurological symptoms due to malignant spinal cord compression. ${ }^{2}$ The bone stability score introduced by Taneichi et al. has been validated to provide an instrument to estimate the probability of collapse of bone metastases. ${ }^{3}$ The usage of a validated scoring tool for the assessment of SBM stability may prevent physicians from overdiagnosis of instability and hence improve QoL 


\section{A Taneichi score for the thoracic spine}

(A)

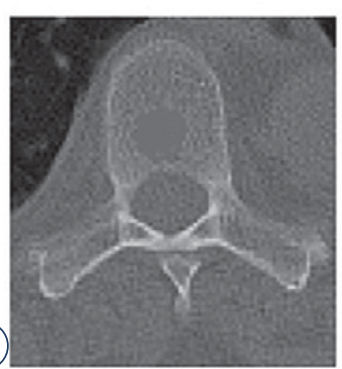

(D)

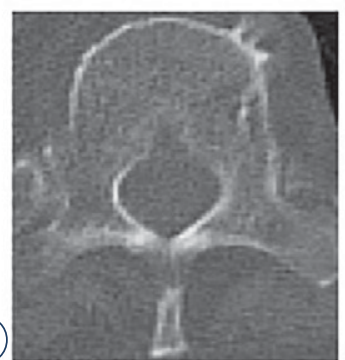

(E)

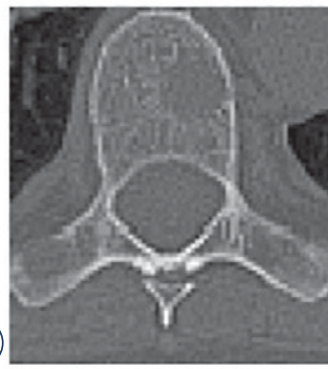

(C)

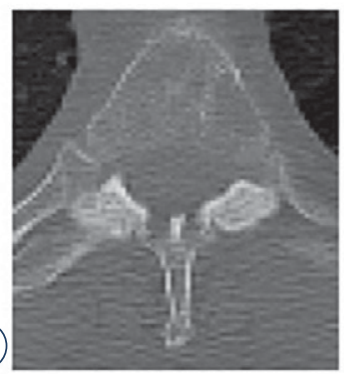

(F)

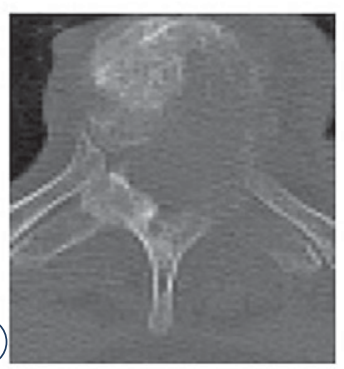

FIGURE 1. Taneichi et al. score for spinal bone metastases of the (A) thoracic and (B) lumbar spine.

\section{B Taneichi score for the lumbar spine}

\section{(A)}

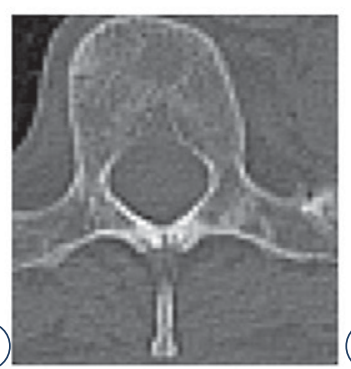

(B)

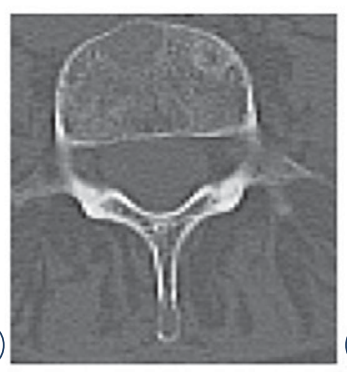

(C)

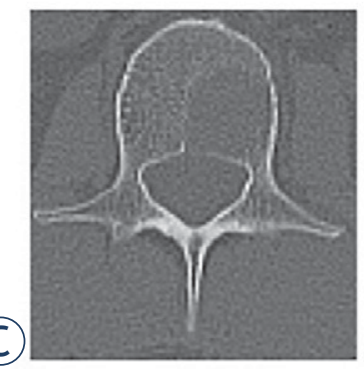

(D)

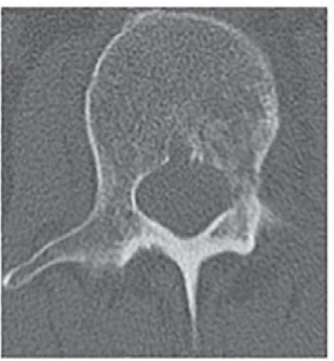

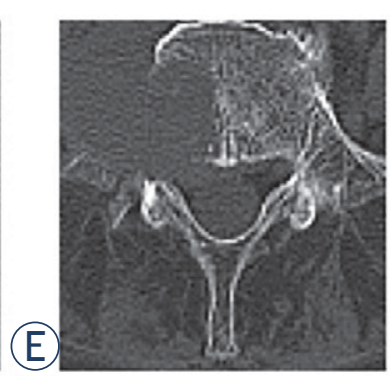

(F)

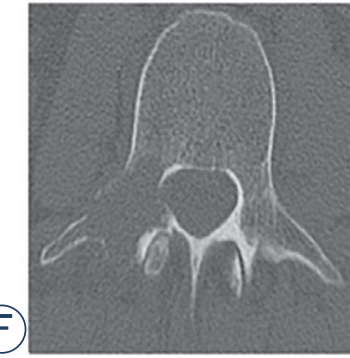

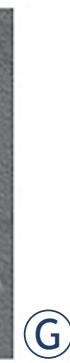

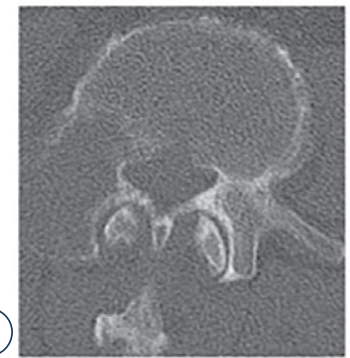

\begin{tabular}{|c|c|c|c|c|c|c|}
\hline \multicolumn{7}{|c|}{ A Taneichi score for the thoracic spine } \\
\hline & A & B & C & D & E & $\mathrm{F}$ \\
\hline $\begin{array}{l}\text { Tumor occupancy } \\
\text { vertebral body }\end{array}$ & $30 \%$ & $60 \%$ & $30 \%$ & $60 \%$ & $30 \%$ & $60 \%$ \\
\hline $\begin{array}{l}\text { Costovertebral joint } \\
\text { destruction }\end{array}$ & No & No & Yes & Yes & Yes & Yes \\
\hline Pedicle destruction & No & No & No & No & Yes & Yes \\
\hline $\begin{array}{l}\text { Posterior elements } \\
\text { destruction }\end{array}$ & No & No & No & No & No & Yes \\
\hline $\begin{array}{l}\text { Predicted probability of } \\
\text { collapse }\end{array}$ & 0.13 & 0.68 & 0.57 & 0.96 & 0.71 & 0.98 \\
\hline
\end{tabular}

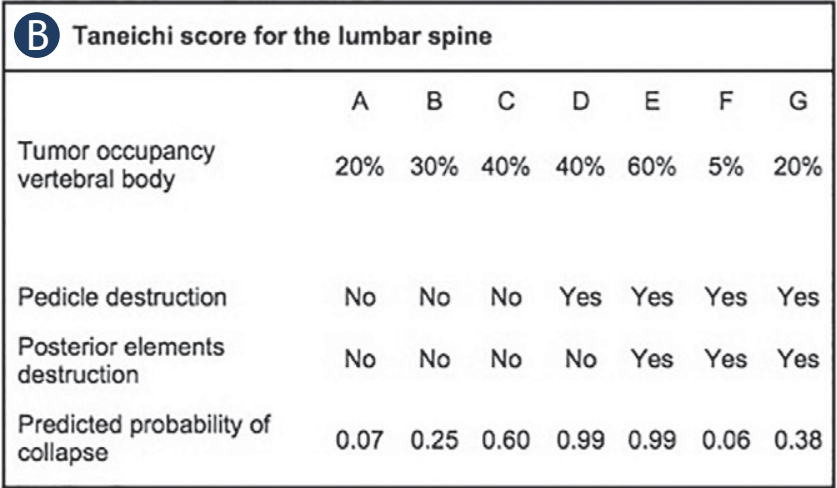


of palliative patients. In earlier studies, we demonstrated that re-ossification and stability can be improved by RT in patients with SBM from nonsmall cell lung cancer (NSCLC), breast cancer, and gynecologic malignancies. ${ }^{4-6}$ We were also able to show that quantification of bone density within metastases can be accurately and easily used to evaluate local response after RT.7,8 However, in two studies with patients suffering from renal cancer and malignant melanoma, stabilization of SBM was not achieved. ${ }^{9,10}$ With this retrospective study, we aimed to analyze survival and clinical outcome of patients with SBM from urothelial cancer as well as to systematically assess bone mineral density (BMD) and stability before and after RT in SBM of these patients.

\section{Patients and methods}

All patients were treated with palliative RT for SBM from histologically diagnosed urothelial cancer at the Department of Radiation Oncology, University Hospital Heidelberg between January 2000 and January 2012. Patients' data including survival status were collected from the institutional cancer registry. Patients received regular clinical followup examinations and computed tomography $(\mathrm{CT})$ scans at 3 and 6 months after RT. All patients were included in the survival analyses.

Nineteen patients with osteolytic SBM of the thoracic or lumbar spine, and a minimum duration of follow-up to treatment of 6 months were included in the stability analyses. For each patient, the most severe metastasis according to the Taneichi et al. score was evaluated in the analysis. Bone metastases were diagnosed by $\mathrm{CT}$, magnetic-resonance imaging or bone-scintigraphy scans. Treatment planning $\mathrm{CT}$ scans were used to assess stability based on the Taneichi et al. score prior to RT; at 3 and 6 months after RT, stability was reassessed using follow-up CT imaging. Osteolytic metastases were rated on a scale from A to G. Subtypes A to $C$ were defined as stable and subtypes $D$ to $G$ as unstable (Figure 1). Height reduction by at least $20 \%$ or visible fracture lines were defining for a new pathological fracture. New partial fractures of a vertebral body did not mandatorily influence the stability score. To assess BMD, we measured Hounsfield units (HU) within the bone metastases by manual regions of interest setting (Figure 2) before RT and 3 months as well as 6 months after RT.

This study was conducted in accordance with the declaration of Helsinki and was approved by

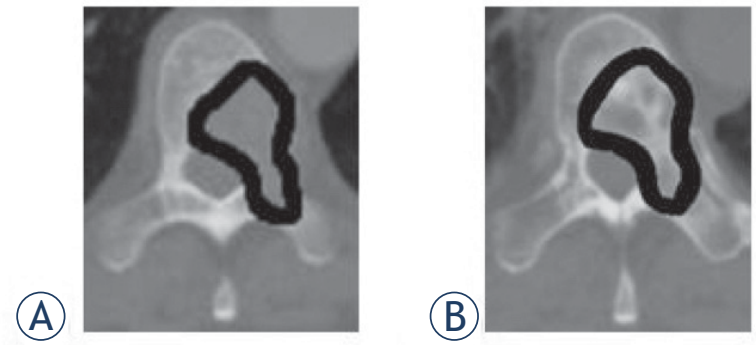

FIGURE 2. Bone mineral density measurement in Hounsfield units by manual region of interest setting (A) before radiotherapy and (B) after radiotherapy within the spinal bone metastases.

the independent ethics committee of the University of Heidelberg on 22 October 2012 (\# S-513/2012). The requirement of informed consent was waived by the ethics committee, due to the retrospective nature of the study.

\section{Patients' characteristics}

RT was performed for osseous instability in 18.5\% $(n=7)$, for pain in $78.9 \%(n=30)$, and for neurological symptoms in $2.6 \%(n=1)$ of patients. Pain and neurological deficits as well as their alterations after RT were recorded during the follow-up examinations, in case they were linked to the irradiated area. Prior fractures affecting the vertebrae in the treated area were detected in the treatment planning $\mathrm{CT}$ scans in 10 patients $(26.3 \%)$. In 1 patient $(3 \%)$, a new fracture was found after RT. Nine patients $(24 \%)$ also had distant metastases in other organs. Most patients were males ( $\mathrm{n}=30,79 \%)$ and had a Karnofsky performance status (KPS) < $80 \%(\mathrm{n}=29 ; 76.3 \%)$. Fourteen patients $(37 \%)$ had received chemotherapy (ChT) with cisplatin and gemcitabine prior to RT. Only 4 patients $(10.5 \%)$ received concomitant bisphosphonates (Table 1).

\section{Radiotherapy}

RT planning was performed based on CT scans, and treatment was performed using a posterior photon field with an energy of $6 \mathrm{MV}$. The planning target volume (PTV) covered the affected vertebral body as well as those immediately above and below. The fractionation schedule was selected individually for each patient, depending on the patient's general state of health, the current staging, response to current therapy and the respective prognosis. The most frequent fractionation schedule was 10 x $3 \mathrm{~Gy}$ (Table 1). 
TABLE 1. Patients' characteristics of all patients with urothelial cancer and spinal bone metastases

\begin{tabular}{|c|c|c|}
\hline \multicolumn{3}{|l|}{ Patients' characteristics } \\
\hline \multicolumn{3}{|l|}{ Age } \\
\hline Median & \multicolumn{2}{|c|}{70 years } \\
\hline Range & \multicolumn{2}{|c|}{$35-82$ years } \\
\hline & $\mathrm{n}$ & $\%$ \\
\hline \multicolumn{3}{|l|}{ Gender } \\
\hline Female & 8 & $21.0 \%$ \\
\hline Male & 30 & $79.0 \%$ \\
\hline \multicolumn{3}{|c|}{ Karnofsky performance status } \\
\hline $40-60 \%$ & 12 & $31.6 \%$ \\
\hline $70 \%$ & 17 & $44.7 \%$ \\
\hline $80 \%$ & 9 & $23.7 \%$ \\
\hline \multicolumn{3}{|l|}{ Histology } \\
\hline Urothelial carcinoma & 38 & $100 \%$ \\
\hline \multicolumn{3}{|l|}{ Localization of metastases } \\
\hline Thoracic & 18 & $47.4 \%$ \\
\hline Lumbar & 20 & $52.6 \%$ \\
\hline \multicolumn{3}{|l|}{ Number of metastases } \\
\hline Single & 15 & $39.5 \%$ \\
\hline Multiple & 23 & $60.5 \%$ \\
\hline \multicolumn{3}{|c|}{ Distant extra-osseous metastases } \\
\hline Overall & 9 & $23.7 \%$ \\
\hline Lungs & 6 & $15.8 \%$ \\
\hline Liver & 5 & $13.2 \%$ \\
\hline Brain & 2 & $5.3 \%$ \\
\hline \multicolumn{3}{|l|}{ Surgical corset } \\
\hline Yes & 6 & $15.8 \%$ \\
\hline No & 32 & $84.2 \%$ \\
\hline \multicolumn{3}{|l|}{ Treatment indications } \\
\hline Instability & 7 & $18.4 \%$ \\
\hline Neurological symptoms & 1 & $2.6 \%$ \\
\hline Pain & 30 & $79.0 \%$ \\
\hline \multicolumn{3}{|l|}{ Radiotherapy schedule } \\
\hline $10 \times 3$ Gy & 24 & $63.2 \%$ \\
\hline $14 \times 2.5 \mathrm{~Gy}$ & 3 & $7.9 \%$ \\
\hline $20 \times 2$ Gy & 11 & $28.9 \%$ \\
\hline \multicolumn{3}{|l|}{ Systemic therapy } \\
\hline Chemotherapy & 14 & $36.8 \%$ \\
\hline Bisphosphonates & 4 & $10.5 \%$ \\
\hline
\end{tabular}

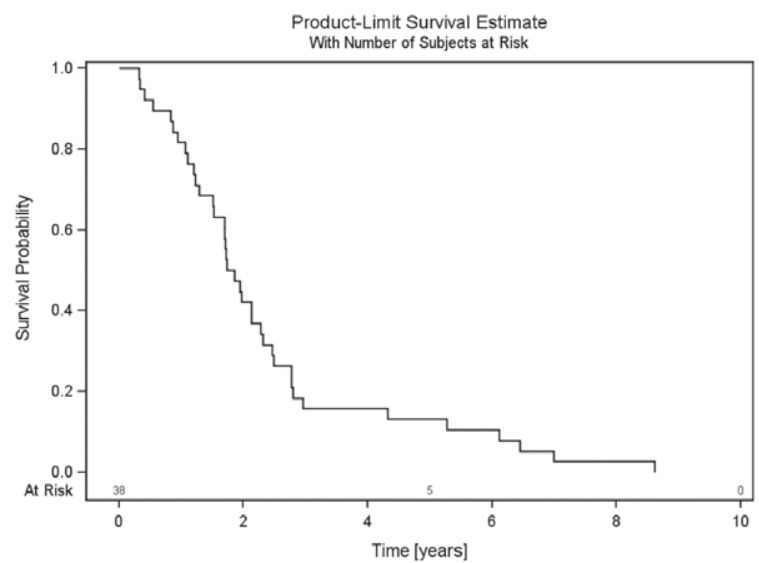

FIGURE 3. Overall survival - survival of all patients with urothelial cancer and spinal bone metastases from first diagnosis of urothelial cancer until death.

\section{Statistical analyses}

Survival was calculated with the Kaplan-Meier method and presented graphically. Patients, who were lost to follow-up, were censored for statistical analyses. Overall survival (OS) was defined as the time from initial diagnosis of urothelial cancer until death and "bone survival" (BS) as the time from first diagnosis of SBM until death. "Radiotherapy survival" (RTS) was defined as the time between start of RT and death. For the assessment of the distribution of Taneichi et al. score subtypes before and at 6 months after RT, Bowker test was used. Kappa statistics were calculated to detect possible asymmetry in the distribution of the Taneichi et al. score over time. BMD was measured in HU. Mean values and the standard deviation (SD) were calculated for BMD before RT as well as at 3 and

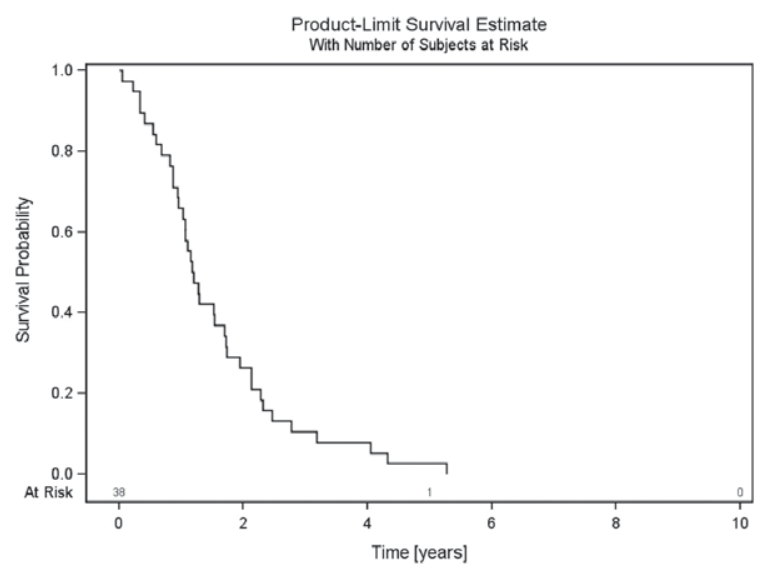

FIGURE 4. Bone survival - survival of all patients with urothelial cancer and spinal bone metastases from first diagnosis of spinal bone metastases until death. 


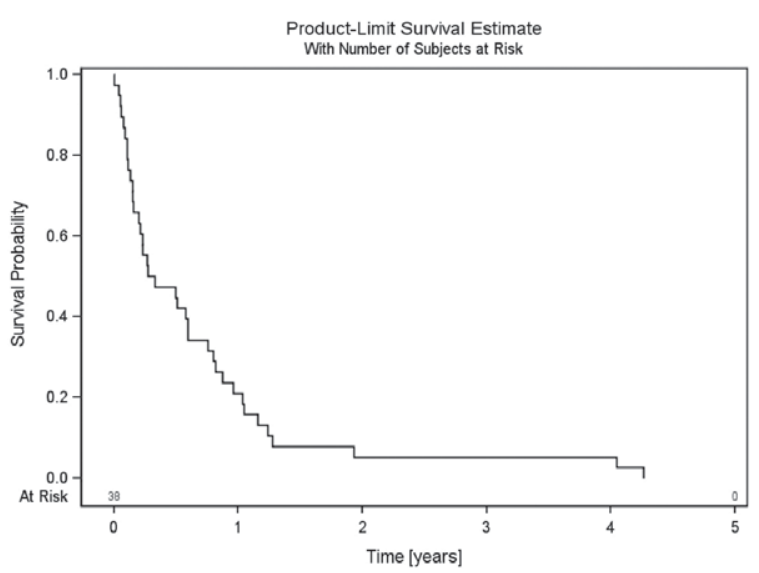

FIGURE 5. Radiotherapy survival - survival of all patients with urothelial cancer and spinal bone metastases from start of palliative radiotherapy until death.

6 months after RT. To statistically analyze the differences in BMD before and after RT, we used the Wilcoxon signed-rank test. A p-value $\leq 0.05$ was considered statistically significant. All statistical analyses were performed with SAS software 9.4 (SAS Institute, Cary, NC, USA).

\section{Results}

The median follow up of all patients was 1.8 years (mean 2.4 years, range $0.3-8.6$ years). All patients died during follow-up. OS after 6 months, 1 year and 2 years was $90 \%, 80 \%$ and $40 \%$, respectively (Figure 3). BS was 85\%, 64\% and 23\% after 6 months, 1 year and 2 years, respectively (Figure 4 ). RTS after 6 months, 1 year and 2 years was $42 \%$, $18 \%$ and $5 \%$, respectively (Figure 5).

Twenty of the 38 patients (52.6\%) were classified as unstable before RT. One of the initially unstable metastases was classified as stable at 3 and 6 months after RT. Additionally, one patient with an initially stable metastasis showed progression of the osteolytic lesion with a new pathological fracture after RT and was classified as unstable after 3 and after 6 months. Overall, 10 of the 19 surviving patients $(52.6 \%)$ were classified as unstable and nine $(47.4 \%)$ were classified as stable after 3 and after 6 months. The evaluation of the distribution of Taneichi et al. subtypes A to G showed no improvement over the course of time. Bowker test confirmed symmetry of the distribution of stability $(\mathrm{p}=1$; Table 2).

Mean calculated metastasis size and mean bone density were $413.8 \mathrm{~mm}^{2} \pm$ SD 253.6 and $110.8 \mathrm{HU} \pm$ SD 63.9 at initial assessment. At 3 months after RT,
TABLE 2. Distribution of subtypes $A$ to $G$ of the Taneichi Score over the course of time (0-6 months)

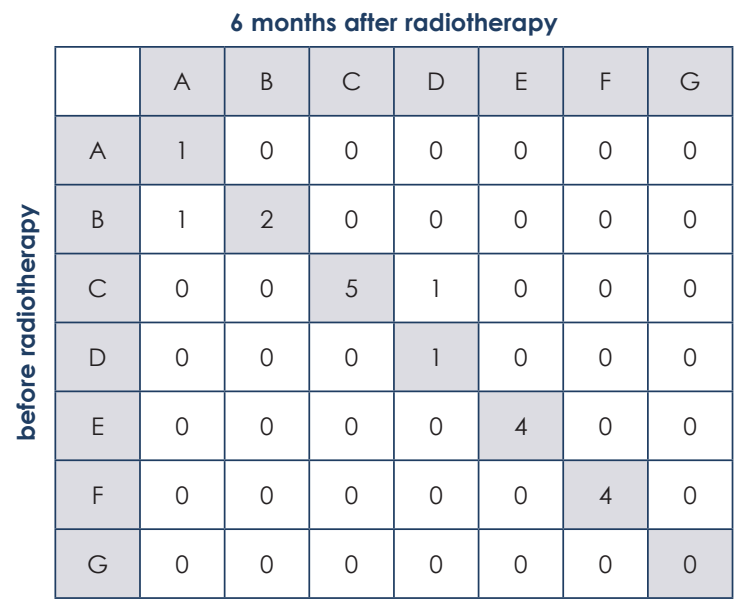

we observed a bone density of $125.7 \mathrm{HU} \pm$ SD 82.6 and after 6 months a bone density of $127.5 \mathrm{HU} \pm$ SD 85.6. The increase of bone density of $25.0 \mathrm{HU} \pm$ $49.7 \mathrm{SD}$ after 3 months and $24.2 \mathrm{HU} \pm 52.2 \mathrm{SD}$ after 6 months was statistically significant $(\mathrm{p}=0.001 ; \mathrm{p}$ $=0.037$ ).

The documented indication for RT was pain in 30 patients and only $26.7 \%(\mathrm{n}=8)$ reported pain relief ( $\geq 2$ points on the visual analogue scale). One patient $(2.6 \%)$ had a neurological deficit with paresthesia of the right laterodorsal thoracic wall and this was unchanged 6 months after RT. None of the patients developed a new neurological deficit.

\section{Discussion}

While survival was comparable to previously published data on patients with metastatic urothelial cancer ${ }^{11}$, we found RTS with $42 \%$ after 6 months to be particularly poor. We believe this to be attributable to patients' extensive morbidity, with a KPS $<80 \%$ in $76.3 \%$ and additional extra-osseous metastases in 9 out of 38 patients, at the initiation of $\mathrm{RT}$, since earlier publications on different ChT regimens in patients with metastatic urothelial cancer reported visceral metastases as well as reduced performance status to be independent predictors of poor outcome. Moreover, these studies also described the presence of bone metastases to be an independent prognostic factor for survival. ${ }^{12,13}$ Nevertheless, there is only a minor impact of local therapy in these patients.

Generally, in patients with osseous metastases of the vertebral column, pain and instability are 
major concerns regularly resulting in reduced QoL. Instability, especially when requiring a prescribed surgical corset, leads to reduced activity in daily life and further impairment of patients' QoL. Taneichi et al. developed a validated scoring system for the probability of vertebral body collapse based on risk factors such as tumor size, costovertebral joint involvement and pedicle destruction. ${ }^{3}$ The use of such a score may aide physicians in the evaluation of vertebral body stability and stabilization after RT. In recent studies on patients with SBM and different primary tumor entities, we found stabilization to be largely dependent on the primary tumor histology. While women with breast cancer and gynecologic malignancies as well as patients with NSCLC may benefit from stabilizing RT, patients with malignant melanoma and renal cancer died before stabilization was achieved. ${ }^{4-6,9,10}$ In our current study, none of the patients with urothelial cancer and initially unstable SBM could be classified as stable 6 months after RT. This poor local response to palliative RT is underlined by our findings regarding BMD. While we were able to demonstrate a statistically significant increase of $25.0 \mathrm{HU} \pm 49.7$ SD $(p=0.001)$ after 3 months and of $24.2 \pm 52.2$ SD $(\mathrm{p}=0.037)$ after 6 months, BMD did not further improve between 3 months and 6 months after RT $(-1.7 \mathrm{HU} \pm 17.6 \mathrm{SD})$. Additionally, increase in HU was small when compared to our previous studies. ${ }^{7,8}$ Furthermore, pain relief was reached in only $26.7 \%$ of the patients with painful SBM. Previous studies on patients with bone metastases from our own institution ${ }^{10,14,15}$ as well as in the international literature $^{16}$ found substantially better response rates. Besides, only $10.5 \%$ of the patients in our study had received concomitant bisphosphonates. A small randomized trial on patients with bladder cancer and bone metastases found pain response, incidence of skeletal-related events and survival to be improved by zoledronic acid. ${ }^{17}$ Therefore, our patients may have benefitted from concomitant bisphosphonate treatment as well. Only one patient had a neurological deficit before RT and there was no improvement during follow-up. We were unable to elucidate, whether this was due to poor response to palliative $\mathrm{RT}$ or due to the duration of symptoms before RT.

Benefit from sole palliative RT of painful or unstable SBM in patients with urothelial cancer is limited. Given the short survival and poor local response, patients should be carefully selected for palliative RT based on their KPS, and longer fractionation schedules, as used in our patients, should be avoided. However, concomitant bisphospho- nates may improve outcome in terms of re-ossification, pain relief and survival.

\section{References}

1. Bianchi $M$, Roghmann $F$, Becker A, Sukumar S, Briganti A, Menon M, et al. Age-stratified distribution of metastatic sites in bladder cancer: A population-based analysis. Can Urol Assoc J 2014; 8: E148-58. doi:10.5489/ cuaj.787

2. Lutz S, Berk L, Chang E, Chow E, Hahn C, Hoskin P, et al. Palliative radiotherapy for bone metastases: an ASTRO evidence-based guideline. Int J Radiat Oncol Biol Phys 2011; 79: 965-76. doi:10.1016/j.ijrobp.2010.11.026

3. Taneichi H, Kaneda K, Takeda N, Abumi K, Satoh S. Risk factors and probability of vertebral body collapse in metastases of the thoracic and lumbar spine. Spine (Phila Pa 1976) 1997; 22: 239-45.

4. Foerster R, Habermehl D, Bruckner T, Bostel T, Schlampp I, Welzel T, et al Spinal bone metastases in gynecologic malignancies: a retrospective analysis of stability, prognostic factors and survival. Radiat Oncol 2014; 9: 194. doi:10.1186/1748-717X-9-194

5. Rief $\mathrm{H}$, Bischof $\mathrm{M}$, Bruckner $\mathrm{T}$, Welzel T, Askoxylakis V, Rieken S, et al. The stability of osseous metastases of the spine in lung cancer - a retrospective analysis of 338 cases. Radiat Oncol 2013; 8: 200. doi:10.1186/1748$717 X-8-200$

6. Schlampp I, Rieken S, Habermehl D, Bruckner T, Förster R, Debus J, et al. Stability of spinal bone metastases in breast cancer after radiotherapy: a retrospective analysis of 157 cases. Strahlenther Onkol 2014; 190: 792-7. doi:10.1007/s00066-014-0651-z

7. Foerster R, Eisele C, Bruckner T, Bostel T, Schlampp I, Wolf R, et al. Bone density as a marker for local response to radiotherapy of spinal bone metastases in women with breast cancer: a retrospective analysis. Radiat Oncol 2015; 10: 62. doi:10.1186/s13014-015-0368-x

8. Rief H, Petersen LC, Omlor G, Akbar M, Bruckner T, Rieken S, et al. The ef fect of resistance training during radiotherapy on spinal bone metastases in cancer patients - a randomized trial. Radiother Oncol 2014; 112: 133-9. doi:0.1016/j.radonc.2014.06.008

9. Bostel T, Förster R, Schlampp I, Wolf R, Serras AF, Mayer A, et al. Stability, prognostic factors and survival of spinal bone metastases in malignant melanoma patients after palliative radiotherapy. Tumori 2016; 102: 156-61. doi:10.5301/tj.5000382

10. Schlampp I, Lang H, Förster R, Wolf R, Bostel T, Bruckner T, et al. Stability of spinal bone metastases and survival analysis in renal cancer after radiotherapy. Tumori 2015; 101: 614-20. doi:10.5301/tj.5000370

11. Abe T, Shinohara N, Harabayashi T, Sazawa A, Maruyama S, Suzuki $\mathrm{S}$, et al. Impact of multimodal treatment on survival in patients with metastatic urothelial cancer. Eur Urol 2007; 52: 1106-13. doi:10.1016/j. eururo.2007.02.052

12. Bellmunt J, Albanell J, Paz-Ares L, Climent MA, Gonzalez-Larriba JL, Carles J, et al. Pretreatment prognostic factors for survival in patients with advanced urothelial tumors treated in a phase $1 / \mathrm{II}$ trial with paclitaxel, cisplatin, and gemcitabine. Cancer 2002; 95: 751-7. doi:10.1002/cncr.10762

13. Saxman SB, Propert KJ, Einhorn LH, Crawford ED, Tannock I, Raghavan D, et al. Long-term follow-up of a phase III intergroup study of cisplatin alone or in combination with methotrexate, vinblastine, and doxorubicin in patients with metastatic urothelial carcinoma: a cooperative group study. J Clin Oncol 1997; 15: 2564-9. doi:10.1200/jco.1997.15.7.2564

14. Habermehl D, Haase K, Rieken S, Debus J, Combs SE. Defining the role of palliative radiotherapy in bone metastasis from primary liver cancer: an analysis of survival and treatment efficacy. Tumori 2011; 97: 609-13. doi:10.1700/989.10720

15. Rief H, Heinhold M, Bruckner T, Schlampp I, Forster R, Welzel T, et al. Quality of life, fatigue and local response of patients with unstable spinal bone metastases under radiation therapy--a prospective trial. Radiat Oncol 2014; 9: 133. doi:10.1186/1748-717X-9-133

16. Chow R, Hoskin P, Chan S, Mesci A, Hollenberg D, Lam H, et al. Efficacy of multiple fraction conventional radiation therapy for painful uncomplicated bone metastases: A systematic review. Radiother Oncol 2017; 122: 323-31. doi:10.1016/j.radonc.2016.12.031

17. Zaghloul MS, Boutrus R, El-Hossieny H, Kader YA, El-Attar I, Nazmy M. A prospective, randomized, placebo-controlled trial of zoledronic acid in bony metastatic bladder cancer. Int J Clin Oncol 2010; 15: 382-9. doi:10.1007/ s10147-010-0074-5 